\title{
Clinical Outcomes of Patient Subgroups in the TANGO II Study
}

Tanaya Bhowmick

Received: July 16, 2020 / Accepted: January 20, 2021 / Published online: February 9, 2021

(C) The Author(s) 2021

\section{ABSTRACT}

Introduction: Meropenem-vaborbactam (MV), a new approved antimicrobial, was developed specifically to be effective treatment for the increasingly prevalent and difficult to treat carbapenem-resistant Enterobacterales (CRE) infections. However, registration phase 3 clinical studies offer limited applicability to daily medical practice as they often focus on indications such as urinary tract infections or skin and soft tissue infections, which generally have patients with fewer comorbid conditions that the typical patients who develops infection with CRE. The more useful studies are pathogen-focused trials which do not exclude the more complicated subjects with conditions such as renal failure, immunocompromised status, or exposure to prior antibiotic therapy.

Methods: The TANGO II study was an open-label investigation of $\mathrm{M}-\mathrm{V}$ compared with the best available treatment (BAT) in hospitalized adults

T. Bhowmick $(\bowtie)$

Department of Medicine, Division of Allergy, Immunology and Infectious Diseases, Rutgers Robert Wood Johnson Medical School, New Brunswick, NJ, USA

e-mail: bhowmita@rwjms.rutgers.edu with a confirmed infection that was known or suspected to be a CRE infection. TANGO II specifically included patients with comorbidities, prior antibiotic therapy, renal failure, and immunocompromised status that are typical in patients with a CRE infection. Interim data analysis indicated that a significant benefit was seen for those patients receiving M-V over BAT. This analysis reports on subsets of TANGO II study patients with multiple comorbidities and high severity of illness, specifically those with prior antibiotic therapy, renal failure, and immunocompromised status. A patient case that highlights particular complexities and challenges of treating patients with CRE infections in the real world is also presented.

Results: Subjects with comorbid conditions had better outcomes when given $\mathrm{M}-\mathrm{V}$ rather than BAT.

Conclusion: $\mathrm{M}-\mathrm{V}$ is a welcome addition to the antibiotic armamentarium for the treatment of severe CRE infections in complicated patients.

Trial Registration: ClinicalTrials.gov identifier NCT02168946.

Keywords: Carbapenem-resistant organisms; Meropenem-vaborbactam; Multidrug-resistant organisms; Vabomere 


\section{Key Summary Points}

Meropenem-vaborbactam (M-V) was developed for and is an effective treatment for serious infections caused by carbapenem-resistant Enterobacterales (CRE).

Patients with multiple comorbidities are not included in clinical trials, but in realworld settings patients with infections caused by CRE are often complex cases with multiple comorbidities.

The TANGO II study compared M-V with the best available treatment for patients with CRE infections, and enrolled patients with multiple comorbidities and high severity of illness, specifically those with prior antibiotic therapy, renal failure, and immunocompromised status.

This subgroup analysis reports on the outcomes of $\mathrm{M}-\mathrm{V}$ treatment in TANGO II patients with prior antibiotic therapy, renal failure, and immunocompromised status. Also, a real-world patient case is presented which highlights the particular complexities and challenges of treating patients with CRE infections.

Subjects with comorbidities, immunocompromised status, and those with impaired renal function who were treated with $\mathrm{M}-\mathrm{V}$ demonstrated better outcomes compared to best available treatment.

\section{DIGITAL FEATURES}

This article is published with digital features, including a summary slide, to facilitate understanding of the article. To view digital features for this article go to https://doi.org/10.6084/ m9.figshare.13615253.

\section{INTRODUCTION}

Antibiotic resistance is a worldwide epidemic. As a result, the World Health Organization (WHO) created a list of the priority pathogens that pharmaceutical research and development should target when developing new antimicrobial agents. Carbapenem-resistant, extendedspectrum beta-lactamase-producing Enterobacterales (CR ESBL) are one of the top three organisms listed in the priority 1 category designated as "critical". The name of "Enterobacteriaceae" pathogens has been updated and are now referred to as Enterobacterales. Moreover, the emergence of Klebsiella pneumoniae carbapenemase (KPC)-2 phenotype across the USA may make many frontline agents inappropriate [1].

Antibiotic-resistant bacteria, in particular carbapenem-resistant Enterobacterales (CRE), are a worldwide public health threat. Various methods have been employed in an effort to combat this issue from mandating antimicrobial stewardship in the USA [2] to providing incentives from the government to develop new antimicrobials and diagnostic testing strategies [3].

Infections with drug-resistant pathogens have high morbidity and mortality $[4,5]$. Initial clinical trials focus on common indications such as skin and soft tissue infection (SSTI) or urinary tract infection (UTI), and include healthier patients with fewer comorbidities. Moreover, most trials focus on disease state rather than pathogen resistance patterns. Once approved, these antibiotics are often used offlabel for more serious infections [6-8].

Unfortunately, patients with CRE infection often have multiple comorbidities and have a higher severity of illness compared to their antibiotic-susceptible counterparts [9-11]. Recruitment for clinical trials evaluating antibiotic efficacy against patients with resistant organisms is difficult, particularly because these patients are severely ill making the consent process more sensitive. Additionally, targeting of specific drug resistance limits the number of qualifying subjects, thereby 
requiring many more patients to be screened to be able to enroll an adequate sample size [12].

These factors make it difficult to study the newly developed drugs in the real-world setting for which they were created. The comorbidities and disease severity of patients contribute complicating factors to treatment approach, influencing pharmacokinetic/pharmacodynamic elements. Patients with renal failure often require antibiotic dose adjustments, immunocompromised patients can be impacted by drug interactions or are at higher risk for adverse events, and prior antibiotic therapy increases the risk for treatment failure [13]. Given these limitations of enrollment into clinical trials, real-world efficacy of medications is often demonstrated by case reports, case series, and registry trials well after the medication comes to market.

\section{METHODS}

TANGO II was a multicenter, randomized, open-label study of meropenem-vaborbactam ( $2 \mathrm{~g} / 2 \mathrm{~g}$ q8h) (M-V) versus best available therapy (BAT) in hospitalized adults with a confirmed range of diagnosed infections requiring administration of intravenous (IV) antibacterial therapy, due to a known or suspected CRE infection [14]. It was a trial conducted in which a new antimicrobial agent was used against infections due to drug-resistant pathogens rather a trial enrolling patients with a specific disease state with infections due to mostly antibiotic-sensitive bacteria [15]. This trial simulated real-world use of the drug in a group of patients who were severely ill. In the comparator group, 64\% received combination therapy-which is often the method of last resort for treating severely ill patients. It took 31 months to enroll and randomize 77 patients for the TANGO II trial. The study was stopped prematurely when interim data analysis indicated that a significant benefit was seen for those patients receiving $\mathrm{M}-\mathrm{V}$ over BAT and it was concluded that randomization was not justified. The details for the clinical trial are described elsewhere [14].

This analysis reports on subsets of TANGO II study patients with multiple comorbidities and high severity of illness, specifically those with prior antibiotic therapy, renal failure, and immunocompromised status. Each comorbid condition was individually assessed with descriptive statistics for this paper. Table 1 describes the baseline demographic and clinical characteristics of TANGO II (mCRE-MITT) patient population [14].

The patient vignette that follows demonstrates the multiple comorbidities and high severity of illness that often accompany the diagnosis of infection with a CRE organism. The patient provided written informed consent to the use of their patient information in the case study.

\section{RESULTS}

\section{Case Example}

A 45-year-old man with childhood agammaglobulinemia was admitted with fevers, fatigue, and transient rash. Laboratory data on admission revealed leukopenia, normal renal function, and an elevated lactate dehydrogenase $(\mathrm{LDH})$ and he was diagnosed with hemophagocytic lymphohistiocytosis (HLH) early in his hospital course. HLH is a disease of excess immune activation which can lead to severe inflammation resulting in multiorgan failure and be life-threatening [16]. He clinically decompensated and was admitted to the intensive care unit with respiratory failure requiring intubation, septic shock requiring vasopressors, and acute renal failure requiring hemodialysis. During his hospital course, he initially received piperacillin-tazobactam from hospital days 1 to 9 which was then changed to meropenem from hospital days 10 to 18 . Cultures performed on tracheal secretions on hospital days 5 and 10 grew a carbapenem-resistant K. pneumoniae (piperacillin-tazobactam minimum inhibitory concentration $[\mathrm{MIC}]>64 /$ $4 \mu \mathrm{g} / \mathrm{ml}$, meropenem MIC $4 \mu \mathrm{g} / \mathrm{ml}$, ceftazidime-avibactam MIC $0.75 \mu \mathrm{g} / \mathrm{ml}$ by E-test, $\mathrm{M}-\mathrm{V}$ disc diffusion zone diameter of $22 \mathrm{~mm}$ ) which was believed to be a colonizer. He subsequently developed bacteremia with positive blood cultures on hospital day 17 with this 
Table 1 Baseline demographic and clinical characteristics of TANGO II (mCRE-MITT) [14]

\begin{tabular}{|c|c|c|c|}
\hline Characteristic & $M-V(n=32)$ & $\operatorname{BAT}(n=15)$ & Total $(n=47)$ \\
\hline Age, mean (SD), years & $63.5(14.1)$ & $60.2(13.0)$ & $62.5(13.7)$ \\
\hline \multicolumn{4}{|l|}{ Age cohort, $n(\%)$} \\
\hline$<65$ years & $17(53.1)$ & $9(60.0)$ & $26(55.3)$ \\
\hline$\geq 65$ years & $8(25.0)$ & $3(20.0)$ & $11(23.4)$ \\
\hline$\geq 75$ years & $7(21.9)$ & $3(20.0)$ & $10(21.3)$ \\
\hline Female gender, $n(\%)$ & $18(56.3)$ & $5(33.3)$ & $23(48.9)$ \\
\hline White race, $n(\%)$ & $28(87.5)$ & $12(80.0)$ & $40(85.1)$ \\
\hline BMI, mean (SD) & $27.9(9.0)$ & $25.8(7.6)$ & $27.2(8.5)$ \\
\hline \multicolumn{4}{|l|}{ Infection type, $n(\%)$} \\
\hline Bacteremia & $14(43.8)$ & $8(53.3)$ & $22(46.8)$ \\
\hline cUTI/AP & $12(37.5)$ & $4(26.7)$ & $16(34.0)$ \\
\hline $\mathrm{HABP} / \mathrm{VABP}$ & $4(12.5)$ & $1(6.7)$ & $5(10.6)$ \\
\hline cIAI & $2(6.3)$ & $2(13.3)$ & $4(8.5)$ \\
\hline \multicolumn{4}{|l|}{ Baseline pathogen, $n(\%)^{\mathrm{a}}$} \\
\hline Klebsiella pneumoniae & $29(90.6)$ & $12(80.0)$ & $41(87.2)$ \\
\hline Escherichia coli & $3(9.4)$ & $1(6.7)$ & $4(8.5)$ \\
\hline Enterobacter cloacae sp. & $1(3.1)$ & $2(13.3)$ & $3(6.4)$ \\
\hline Proteus mirabilis & $0(0)$ & $2(13.3)$ & $2(4.3)$ \\
\hline Serratia marcescens & $1(3.1)$ & $1(6.7)$ & $2(4.3)$ \\
\hline Enrolled as confirmed CRE, $n(\%)$ & $23(71.9)$ & $14(93.3)$ & $37(78.7)$ \\
\hline Enrolled as suspected CRE, $n$ (\%) & $9(28.1)$ & $1(6.7)$ & $10(21.3)$ \\
\hline \multicolumn{4}{|l|}{ Creatinine clearance, $\mathrm{ml} / \mathrm{min}, n(\%)$} \\
\hline$\geq 50$ & $24(75.0)$ & $9(60.0)$ & $33(70.2)$ \\
\hline $30-49$ & $4(12.5)$ & $2(13.3)$ & $6(12.8)$ \\
\hline $20-29$ & $1(3.1)$ & $2(13.3)$ & $3(6.4)$ \\
\hline$<20$ & $2(6.3)$ & $0(0)$ & $2(4.3)$ \\
\hline Missing & $1(3.1)$ & $2(13.3)$ & $3(6.4)$ \\
\hline \multicolumn{4}{|l|}{ Charlson comorbidity index, $n(\%)$} \\
\hline$\leq 2$ & $4(12.5)$ & $1(6.7)$ & $5(10.6)$ \\
\hline $3-4$ & $3(9.4)$ & $2(13.4)$ & $5(10.6)$ \\
\hline 5 & $11(34.4)$ & $1(6.7)$ & $12(25.5)$ \\
\hline$\geq 6$ & $14(43.8)$ & $11(73.3)$ & $25(53.2)$ \\
\hline Diabetes mellitus, $n(\%)$ & $12(37.5)$ & $7(46.7)$ & $19(40.4)$ \\
\hline
\end{tabular}


Table 1 continued

\begin{tabular}{llll}
\hline Characteristic & M-V $(\boldsymbol{n}=\mathbf{3 2})$ & BAT $(\boldsymbol{n}=\mathbf{1 5})$ & Total $(\boldsymbol{n}=\mathbf{4 7})$ \\
\hline SIRS, $n$ (\%) & $15(46.9)$ & $6(40.0)$ & $21(44.7)$ \\
ICU admission, $n$ (\%) & $5(15.6)$ & $3(20.0)$ & $8(17.0)$ \\
Immunocompromised $^{\mathrm{b}}, n(\%)$ & $11(34.4)$ & $8(53.3)$ & $19(40.4)$ \\
Prior antibiotic failure $^{\mathrm{c}}, n(\%)$ & $9(28.1)$ & $0(0)$ & $9(19.1)$ \\
\hline
\end{tabular}

Adapted and reused with permission under open access Creative Commons Attribution-NonCommercial 4.0 International License (http://creativecommons.org/licenses/by-nc/4.0/) from Wunderink et al. [14]

$B A T$ best available therapy, $B M I$ body mass index, $C I A I$ complicated intra-abdominal infection, $C R E$ carbapenem-resistant Enterobacteriaceae, $c U T I / A P$ complicated urinary tract infection/acute pyelonephritis, $H A B P / V A B P$ hospital-acquired bacterial pneumonia/ventilator-associated bacterial pneumonia, ICU intensive care unit, $m C R E-M I T T$ microbiologic carbapenem-resistant Enterobacteriaceae modified intent-to-treat, $M-V$ meropenem-vaborbactam, $S D$ standard deviation, SIRS systemic inflammatory response syndrome

${ }^{a}$ Baseline pathogens listed occurred in 2 or more patients

b Receipt of immunosuppressive medications or bone marrow ablative chemotherapy, underlying lymphoma or leukemia (not in remission), previous transplantation, splenectomy, or presence of neutropenia

c Clinical evidence of prior antimicrobial failure as ascertained by the study investigator at screening and randomization

same organism. His hospital course was complicated by disseminated intravascular coagulation, severe hepatitis, encephalopathy, acute punctate infarct in the left cerebellum, aspergillosis of the scrotum, and necrotizing pancreatitis associated with fluid collections of which cultures also grew the same carbapenemresistant K. pneumoniae, now resistant to $\mathrm{M}-\mathrm{V}$. He was treated with multiple antibiotics and eventually changed to ceftazidime-avibactam for completion of therapy. He received dexamethasone, etoposide, and intrathecally administered methotrexate for HLH with repeat BM biopsy showing no disease. He was discharged after a 5-month hospital stay to a rehabilitation center.

This case demonstrates the complexity of patients who develop CRE infections. Some have an underlying immunocompromising condition such as cancer, organ transplant, or receipt of immunosuppressants and then clinically deteriorate with sepsis due to an infection by a CRE resulting in organ dysfunction or organ failure.

Subgroup analyses of patients without prior antibiotic therapy, immunocompromised state, and renal insufficiency in the TANGO II study suggest that at both the end of therapy (EOT) and test of cure (TOC $=\mathrm{EOT}+7$ days) time points, $\mathrm{M}-\mathrm{V}$ had higher clinical cure rates in addition to decreased 28-day all-cause mortality when compared to BAT.

This is the only report to our knowledge to evaluate subgroups of patients with carbapenem-resistant organisms.

\section{Prior Antibiotic Therapy}

Most patients who develop infections with CRE have been exposed to multiple previous antibiotics as in the previously described case. Some have failed therapy, by either developing resistance to the treatment administered or lack of clinical response despite antibiotic administration, may have an empiric change of antibiotic agents. The patient case described earlier had improved with empiric treatment with a less broad-spectrum antibiotic agent; however, he subsequently developed septic shock for which a change in antibiotics was warranted. 
BAT was chosen at the discretion of the study investigator and consisted of antibiotic therapy either alone or in combination of carbapenems, aminoglycosides, colistin, polymixin B, tigecycline or monotherapy with ceftazidime-avibactam (C-A). Prior antibiotic failure was determined by the study investigator at the time of enrollment. Of the 38 patients who were analyzed, 23 received $\mathrm{M}-\mathrm{V}$ and 15 received BAT.

Of the patients who were not categorized as prior antibiotic failure in the TANGO II trial, those who received primary therapy with $\mathrm{M}-\mathrm{V}$ had better outcomes compared with those who received BAT within the microbiologic-CRE modified intent-to-treat (mCRE-MITT) group [17]. The mCRE-MITT was defined as patients who received at least one dose of study drug and had culture confirmation of a baseline isolate that was CRE (Table 2) [17].

The baseline pathogen was K. pneumoniae in approximately $90 \%$ of these patients, the same pathogen isolated in our patient case. Clinical cure at TOC was higher $(69.6 \%$ vs. $26.7 \%$, absolute difference +42.9 ) in the $\mathrm{M}-\mathrm{V}$ group compared to BAT. This difference continued through the evaluation of clinical cure at EOT
(82.6\% vs. $33.3 \%$ ). Similarly, M-V showed better efficacy than BAT when evaluated for microbiologic cure at EOT $(82.6 \%$ vs. $40 \%)$, microbiologic cure at TOC (69.9\% vs. $33.3 \%)$, and 28 -day mortality (4.3\% vs. $33.3 \%)$. This analysis supports the clinical application of $\mathrm{M}-\mathrm{V}$ over BAT in patients who have not failed prior antibiotic therapy.

\section{Immunocompromised}

Among patients with solid tumors or hematologic malignancy and CRE infections, mortality rates are extremely high-up to $60 \%[18,19]$. The patient described in our case did not have a malignancy; however, it is evident that his immune system was compromised as demonstrated by the development of disseminated aspergillus infection, which is rarely seen in the immunocompetent host [20]. Unlike most phase 3 studies of new antimicrobials, TANGO II included immunocompromised patients.

Immunocompromised status was defined as underlying active leukemia, lymphoma, prior

Table 2 Efficacy results in patients without prior antimicrobial failure in the mCRE-MITT population [17]

\begin{tabular}{|c|c|c|c|}
\hline $\begin{array}{l}\text { Efficacy endpoints (mCRE- } \\
\text { MITT) }\end{array}$ & $\begin{array}{l}\text { Meropenem-vaborbactam } \\
(n=23)\end{array}$ & $\begin{array}{l}\text { Best available therapy } \\
(n=15)\end{array}$ & $\begin{array}{l}\text { Absolute difference } \\
(95 \% \mathrm{CI})\end{array}$ \\
\hline Clinical cure at TOC & $16(69.6)$ & $4(26.7)$ & $\begin{array}{c}+42.9(+13.7 \\
\text { to }+72.1)\end{array}$ \\
\hline Clinical cure at EOT & $19(82.6)$ & $5(33.3)$ & $\begin{array}{c}+49.3(+20.8 \\
\text { to }+77.7)\end{array}$ \\
\hline Microbiologic cure $^{a}$ at EOT & $19(82.6)$ & $6(40.0)$ & $\begin{array}{c}+42.6(+13.4 \\
\text { to }+71.8)\end{array}$ \\
\hline Microbiologic cure ${ }^{a}$ at TOC & $16(69.6)$ & $5(33.3)$ & $\begin{array}{c}+36.2(+5.9 \\
\text { to }+66.6)\end{array}$ \\
\hline Day-28 mortality & $1(4.3)$ & $5(33.3)$ & $\begin{array}{l}-29.0(-54.3 \\
\text { to }-3.7)\end{array}$ \\
\hline
\end{tabular}

Reused with permission under Creative Commons Attribution-NonCommercial 4.0 International License (http:// creativecommons.org/licenses/by-nc/4.0/) from Bassetti et al. [17]. https://doi.org/10.1007/s12325-019-00981-y $C I$ confidence intervals, EOT end of therapy, $m C R E-M I T T$ microbiologic carbapenem-resistant Enterobacteriaceae modified intent-to-treat, $T O C$ test of cure

${ }^{a}$ Microbiologic cure was defined as microbial eradication or presumed eradication 
transplant or splenectomy on medical history, any active receipt of immunosuppressive drugs including selective immunosuppressants, calcineurin inhibitors, or high-dose systemic steroids (equivalent to more than $20 \mathrm{mg}$ /day of prednisone for more than 2 weeks) or neutropenia (absolute neutrophil count [ANC] less than 1000 cells $/ \mathrm{mm}^{3}$ ) at any point during the study period.

Of the 50 subjects who had a baseline pathogen (m-MITT population), 19 (38.0\%) were immunocompromised: four leukemia/lymphoma, five medication, and 10 transplant cases. Forty-three of these 50 patients had an identified baseline pathogen which was CRE (mCRE-MITT); 18 (41.9\%) were categorized as immunocompromised. The most common infection types among immunocompromised subjects (mCRE-MITT) were bacteremia $(11 / 18$, $61.1 \%)$, cUTI/AP $(3 / 18,16.7 \%), \operatorname{HABP} / \operatorname{VABP}(2 /$ $18,11.1 \%)$, and CIAI $(2 / 18,11.1 \%)$.

Clinical cure rates for mCRE-MITT immunocompromised subjects were higher for those in the M-V arm compared to the BAT arm with an absolute increase of $47.5 \%$ at EOT and $70 \%$ at TOC. On ad hoc analysis, the increase in cure rate at TOC achieved statistical significance (95\% CI 41.6-98.4, $p<0.0001)$. Rates of microbial cure (defined as either microbial eradication or presumed eradication) for the 18 immunocompromised subjects were higher for those in the $\mathrm{M}-\mathrm{V}$ arm vs. BAT arm (absolute increase of $47.5 \%$ at EOT and $70 \%$ at TOC). The all-cause mortality at day 28 was lower for those patients who received $\mathrm{M}-\mathrm{V}$ compared to those who received BAT (20\% vs. $37.5 \%)$. This was associated with an absolute risk reduction (mortality) of $17.5 \%$ and relative risk reduction of $46.7 \%$. Table 3 shows the efficacy endpoints of the mCRE-MITT patient population, including those with immunocompromised and renal insufficiency [14].

In addition, there were no marked treatment adverse events (AE) noted. Among immunocompromised subjects, $\mathrm{M}-\mathrm{V}$ was associated with fewer adverse events compared to BAT $(84.6 \%$ vs. $100 \%)$, drug-related AEs (30.8\% vs. $40.0 \%)$, serious AEs (38.5\% vs. 50.0\%), discontinuations of study drug or study due to AEs (15.4\% vs. $30.0 \%$ ), and renal-related AEs (7.7\% vs. $40.0 \%$ ).

\section{Renal Insufficiency}

Most patients with sepsis have some degree of acute kidney injury sometimes requiring hemodialysis as exemplified in the case previously described. Patients with decreased kidney function have an increased risk for hospital-related infection in a linear distribution [21]. In addition, renal insufficiency impacts accurate dosing of medications and limits use of agents such as colistimethate and polymixin B which have adverse side effects of renal failure $[22,23]$. Among the 43 subjects who had a baseline CRE organism and were included in the mCRE-MITT population, nine $(20.9 \%)$ subjects had a baseline creatinine clearance $(\mathrm{CrCl})$ less than $50 \mathrm{ml} /$ min. Dose reduction of $\mathrm{M}-\mathrm{V}$ was given on the basis of $\mathrm{CrCl}$. If the $\mathrm{CrCl}$ was less than $50 \mathrm{ml} /$ min, a standard dose of $2 \mathrm{~g}-2 \mathrm{~g}$ was administered every $8 \mathrm{~h}$. If the $\mathrm{CrCl}$ was between 30 and $49 \mathrm{ml} / \mathrm{min}$, the dose was reduced by half to $1 \mathrm{~g}-1 \mathrm{~g}$ every $8 \mathrm{~h}$. The dose was reduced to onethird of the standard dose with $\mathrm{CrCl}$ between 20 and $29 \mathrm{ml} / \mathrm{min}$, to one-sixth of the dose for CrCL between 10 and 19, and finally to onetwelfth of the dose to $500 \mathrm{mg}-500 \mathrm{mg}$ every $24 \mathrm{~h}$. Although hemodialysis patients could be enrolled in the study, those patients who required continuous renal replacement therapy (CRRT) were not eligible for study participation. Since CRRT is more physiologic compared to hemodialysis, we do not see this as a negative to the study exclusion.

Subjects with renal impairment with a $\mathrm{CrCl}$ less than $50 \mathrm{ml} / \mathrm{min}$ had lower clinical cure rates across all infection types at EOT compared with subjects with normal renal function, though there were only nine patients with impaired renal function in the mCRE-MITT cohort. Two of the five patients in the $\mathrm{M}-\mathrm{V}$ group and one of the four in the BAT group achieved both clinical cure at the end of therapy and microbiologic eradication.

Patients with renal insufficiency had lower clinical cure rates across all infection times at the end of IV therapy compared to those with normal renal function regardless of treatment type (M-V $40 \%$ vs. $68.2 \%$; BAT $25 \%$ vs. $44.4 \%$ ). In terms of AEs, M-V had similar rates of treatment emergent adverse events (TEAE) compared 


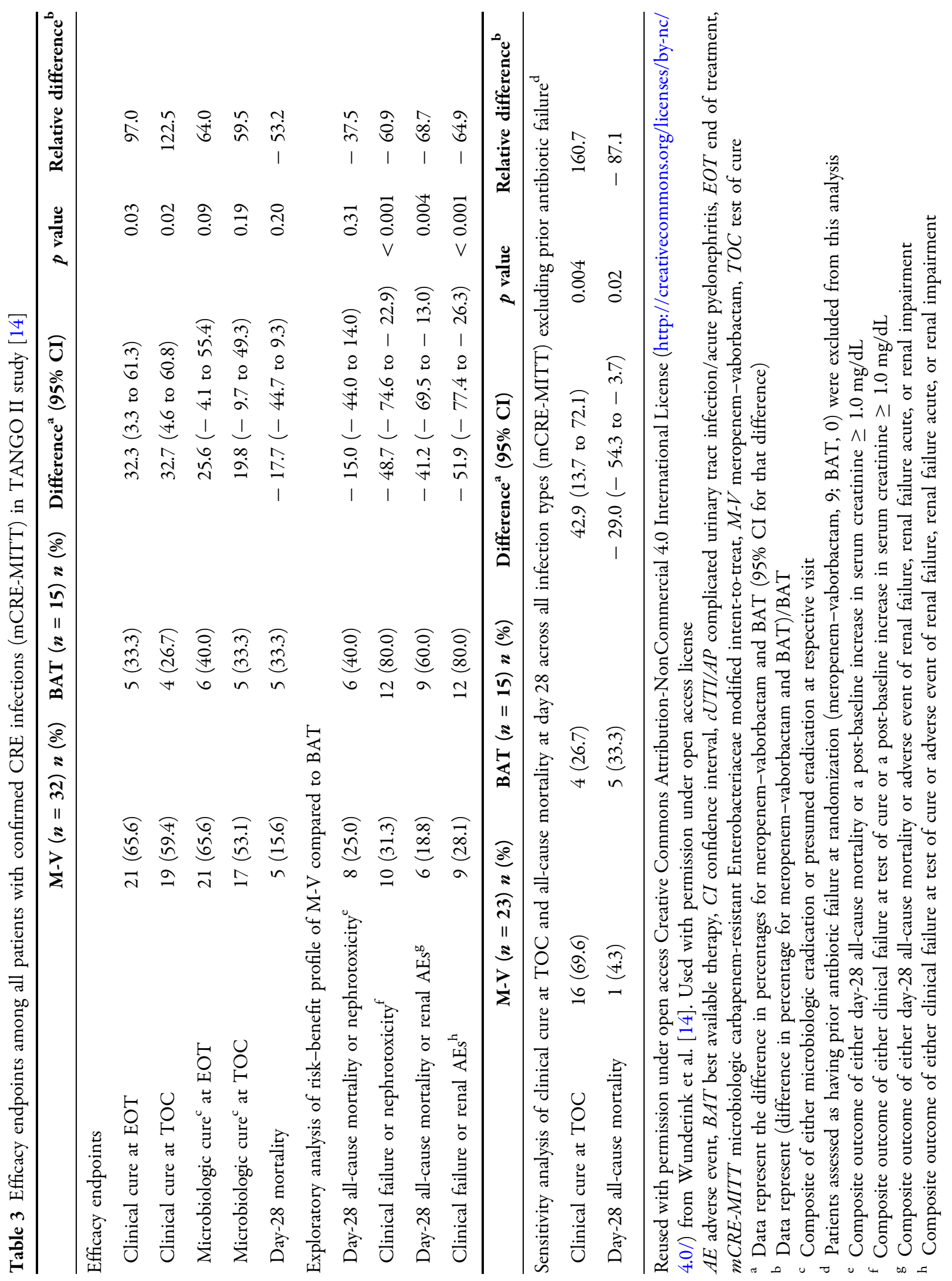


to BAT in the 19 patients who had renal insufficiency ( $8 / 10$ vs. $8 / 9)$.

There is no increased safety signal associated with $\mathrm{M}-\mathrm{V}$ in patients with renal impairment.

$\mathrm{M}-\mathrm{V}$ is a safe and effective treatment for serious gram-negative infections in renally impaired patients when the dose is adjusted on the basis of creatinine clearance.

\section{DISCUSSION}

The newer $\beta$-lactam $/ \beta$-lactamase agents $(\mathrm{M}-\mathrm{V}$, C-A) have been much needed additions to the antibiotic armamentarium for the treatment of carbapenem-resistant infections. Physicians can more readily avoid use of colistin which has inherent difficulties for appropriate dosing, adverse effects most concerning of which is acute kidney injury [24]. At the Clinical Laboratory Standards Institute (CLSI) meeting in June 2019, the colistin breakpoints were tentatively revised such that no MIC was considered susceptible [25]. In addition, the newer agents have potentially fewer toxicities than other classes of drugs used to treat multidrug-resistant organisms.

Indications for UTIs and SSTIs have been most commonly used in clinical trials evaluating newer antimicrobials, and owing to the higher prevalence of these types of infections, recruiting sufficient number of patients is easier. Additionally, strict exclusion criteria result in enrolled patients with minimal or no comorbidities, less severe infections, or other serious health conditions. Therefore, the true niche for these broad-spectrum agents is not represented, including those with acute renal failure, prior antibiotic therapy without failure, immunocompromised status, or patients with malignancies. In contrast, the TANGO II trial was different, and investigators enrolled patients with renal insufficiency and on hemodialysis and accepted patients with malignancies, organ and stem cell transplantation, and receipt of immunosuppressive medications.

There are not many pathogen-directed clinical trials [26-29]. Most have small sample sizes and study drug is compared to BAT similar to the TANGO II trial. Despite the less stringent exclusion criteria in terms of comorbid conditions, the enrollment of patients with suspected or proven CRE infections remained slow. Despite almost 3 years of recruitment for the clinical trial, only 77 patients were enrolled-a testament to the difficulty of recruitment in pathogen specific trials. TANGO II was stopped early because the data safety monitoring board felt the superior efficacy of $M-V$ vs. BAT on interim analysis meant it was inappropriate to continue with the study.

This analysis has some limitations. It is a post hoc evaluation of 77 patients into specific subgroups resulting in even smaller numbers of patients to examine. With fewer subjects, matched controls and regression analysis are not possible. Therefore, the conclusions that are drawn are more observational in nature. Future prospective studies are needed to better evaluate each subgroup; however, this will be a challenging endeavor as demonstrated by the mere difficulty with recruitment for TANGO II which, although strict in terms of recruitment criteria based on pathogen, encompassed all the subgroups.

With newer agents, we still need to be vigilant and cognitive of their limitations. Already there have been reports of the development of treatment-emergent resistance to ceftazidime-avibactam [7, 30-34]. Athans et al. [35] described a liver transplant recipient who developed an infection with a CRE K. pneumoniae which was initially susceptible to C-A, subsequently developed resistance to C-A while on therapy, and eventually received $\mathrm{M}-\mathrm{V}$ which allowed a second liver transplant [18].

\section{CONCLUSION}

$\mathrm{M}-\mathrm{V}$, which was frequently used as monotherapy in the TANGO II trial, has been shown to be effective for the treatment of serious infections caused by carbapenem-resistant Enterobacterales-the purpose for which $\mathrm{M}-\mathrm{V}$ was developed. It has been shown to have better outcomes when compared to receipt of BAT, with an even lower incidence of adverse events. Moreover, this subgroup analysis demonstrates effectiveness in patients who have do not have 
previous antibiotic failure, are immunocompromised, and those with impaired renal function.

\section{ACKNOWLEDGEMENTS}

Funding. No funding was received in the writing of this manuscript. The journal's Rapid Service fee was fully funded by Melinta Therapeutics. Tanaya Bhowmick received no payment from Melinta Therapeutics.

Editorial Assistance. The author wishes to thank Glenn Tillotson and Nicolette Theriault at GST Micro for their assistance throughout the editing and publication of this manuscript.

Authorship. All named authors meet the International Committee of Medical Journal Editors (ICMJE) criteria for authorship for this article, take responsibility for the integrity of the work as a whole, and have given their approval for this version to be published.

Disclosures. Tanaya Bhowmick has nothing to disclose.

Compliance with Ethics Guidelines. Written informed consent for the patient information used in the case study to be published was provided by the patient.

Data Availability. Data sharing is not applicable to this article as it is proprietary.

Open Access. This article is licensed under a Creative Commons Attribution-NonCommercial 4.0 International License, which permits any non-commercial use, sharing, adaptation, distribution and reproduction in any medium or format, as long as you give appropriate credit to the original author(s) and the source, provide a link to the Creative Commons licence, and indicate if changes were made. The images or other third party material in this article are included in the article's Creative Commons licence, unless indicated otherwise in a credit line to the material. If material is not included in the article's Creative Commons licence and your intended use is not permitted by statutory regulation or exceeds the permitted use, you will need to obtain permission directly from the copyright holder. To view a copy of this licence, visit http://creativecommons.org/licenses/by$\mathrm{nc} / 4.0 /$.

\section{REFERENCES}

1. Spellberg B, Bonomo RA. Editorial commentary: ceftazidime-avibactam and carbapenem-resistant Enterobacteriaceae: "we're gonna need a bigger boat." Clin Infect Dis. 2016;63(12):1619-21.

2. Joint Commission on Hospital Accreditation. Approved: new antimicrobial stewardship standard. Jt Comm Perspect. 2016;36(7):1-8.

3. Presidential Advisory Council on Combating Antibiotic-Resistant Bacteria (PACCARB). 2020. https://www.hhs.gov/ash/advisory-committees/ paccarb/about-paccarb/index.html. Accessed $8 \mathrm{Jul}$ 2019.

4. Burnham JP, Olsen MA, Kollef MH. Re-estimating annual deaths due to multidrug-resistant organism infections. Infect Control Hosp Epidemiol. 2018;40(1):112-3.

5. Cassini A, Högberg LD, Plachouras D, et al. Attributable deaths and disability-adjusted lifeyears caused by infections with antibiotic-resistant bacteria in the EU and the European Economic Area in 2015: a population-level modelling analysis. Lancet Infect Dis. 2019;19(1):56-66.

6. Pani A, Colombo F, Agnelli F, et al. Off-label use of ceftaroline fosamil: a systematic review. Int J Antimicrobial Agents. 2019;54(5):562-71.

7. Shields RK, Potoski BA, Haidar G, et al. Clinical outcomes, drug toxicity, and emergence of ceftazidime-avibactam resistance among patients treated for carbapenem-resistant Enterobacteriaceae infections. Clin Infect Dis. 2016;63(12):1615-8.

8. Vickery SB, McClain D, Wargo KA. Successful use of ceftolozane-tazobactam to treat a pulmonary exacerbation of cystic fibrosis caused by multidrug-resistant Pseudomonas aeruginosa. Pharmacotherapy. 2016;36(10):e154-9.

9. Ben-David D, Kordevani R, Keller N, et al. Outcome of carbapenem resistant Klebsiella pneumoniae bloodstream infections. Clin Microbiol Infect. 2012;18(1):54-60. 
10. Borer A, Saidel-Odes L, Riesenberg K, et al. Attributable mortality rate for carbapenem-resistant Klebsiella pneumoniae bacteremia. Infect Control Hosp Epidemiol. 2015;30(10):972-6.

11. Patel G, Huprikar S, Factor SH, Jenkins SG, Calfee DP. Outcomes of carbapenem-resistant Klebsiella pneumoniae infection and the impact of antimicrobial and adjunctive therapies. Infect Control Hosp Epidemiol. 2015;29(12):1099-106.

12. Jorgensen SCJ, Rybak MJ. Pathogen-specific clinical trials: a new paradigm in clinical trials for multidrug-resistant organisms. Infect Dis Therapy. 2018;7(4):401-5.

13. Peeters P, Ryan K, Karve S, et al. The impact of initial antibiotic treatment failure: real-world insights in patients with complicated, health careassociated intra-abdominal infection. Infect Drug Resist. 2019;12:329-43.

14. Wunderink RG, Giamarellos-Bourboulis EJ, Rahav $\mathrm{G}$, et al. Effect and safety of meropenem-vaborbactam versus best-available therapy in patients with carbapenem-resistant Enterobacteriaceae infections: the TANGO II randomized clinical trial. Infect Dis Therapy. 2018;7(4):439-55.

15. Wagenlehner FM, Sobel JD, Newell P, et al. Ceftazidime-avibactam versus doripenem for the treatment of complicated urinary tract infections, including acute pyelonephritis: RECAPTURE, a phase 3 randomized trial program. Clin Infect Dis. 2016;63(6):754-62.

16. Hayden A, et al. Hemophagocytic syndromes (HPSs) including hemophagocytic lymphohistiocytosis (HLH) in adults: a systematic scoping review. Blood Rev. 2016;30(6):411-20.

17. Bassetti M, et al. Efficacy and safety of meropenemvaborbactam versus best available therapy for the treatment of carbapenem-resistant enterobacteriaceae infections in patients without prior antimicrobial failure: a post hoc analysis. Adv Ther. 2019;36(7):1771-7.

18. Alexander EL, Loutit J, Tumbarello M, et al. Carbapenem-resistant enterobacteriaceae infections: results from a retrospective series and implications for the design of prospective clinical trials. Open Forum Infect Dis. 2017;4(2):ofx063.

19. Satlin MJ, Jenkins SG, Walsh TJ. The global challenge of carbapenem-resistant Enterobacteriaceae in transplant recipients and patients with hematologic malignancies. Clin Infect Dis. 2014;58(9): 1274-83.

20. Mandell D. Bennett's principles and practice of infectious diseases. In: Bennett JE, Dolin R, Blaser
MJ, editors. Principles and practice of infectious diseases, 9th edn, vol 3105-3106. Philadelphia: Elsevier; 2019.

21. Dalrymple LS, et al. The risk of infection-related hospitalization with decreased kidney function. Am J Kidney Dis. 2012;59(3):356-63.

22. Tsuji BT, et al. International Consensus Guidelines for the Optimal Use of the Polymyxins: Endorsed by the American College of Clinical Pharmacy (ACCP), European Society of Clinical Microbiology and Infectious Diseases (ESCMID), Infectious Diseases Society of America (IDSA), International Society for Anti-infective Pharmacology (ISAP), Society of Critical Care Medicine (SCCM), and Society of Infectious Diseases Pharmacists (SIDP). Pharmacother J Human Pharmacol Drug Therapy. 2019;39(1):10-39.

23. Livornese LL, et al. Use of antibacterial agents in renal failure. Infect Dis Clin North Am. 2004;18(3): 551-79.

24. Miano TA, et al. Attributable risk and time course of colistin-associated acute kidney injury. Clin J Am Soc Nephrol. 2018;13(4):542-50.

25. Presentation BPWG (Break Point Working Group). CLSI (Clinical Laboratory Standards Institute). Dallas; 2019.

26. Carmeli Y, et al. Ceftazidime-avibactam or best available therapy in patients with ceftazidime-resistant Enterobacteriaceae and Pseudomonas aeruginosa complicated urinary tract infections or complicated intra-abdominal infections (REPRISE): a randomised, pathogen-directed, phase 3 study. Lancet Infect Dis. 2016;16(6):661-73.

27. McKinnell JA, et al. Plazomicin for infections caused by carbapenem-resistant Enterobacteriaceae. N Engl J Med. 2019;380(8):791-3.

28. Motsch J, et al. RESTORE-IMI 1: a multicenter, randomized, double-blind trial comparing efficacy and safety of imipenem/relebactam vs colistin plus imipenem in patients with imipenem-nonsusceptible bacterial infections. Clin Infect Dis. 2020;70(9):1799-1808.

29. Paul $\mathrm{M}$, et al. Colistin alone versus colistin plus meropenem for treatment of severe infections caused by carbapenem-resistant Gram-negative bacteria: an open-label, randomised controlled trial. Lancet Infect Dis. 2018;18(4):391-400.

30. Shields RK, et al. Pneumonia and renal replacement therapy are risk factors for ceftazidime-avibactam treatment failures and resistance among patients with carbapenem-resistant Enterobacteriaceae 
infections. Antimicrob Agents Chemother. 2018;62(5):e02497-e2517.

31. Shields RK, et al. Emergence of ceftazidime-avibactam resistance and restoration of carbapenem susceptibility in Klebsiella pneumoniae carbapenemaseproducing $K$. pneumoniae: a case report and review of literature. Open Forum Infect Dis. 2017;4(3): ofx101.

32. Tumbarello $M$, et al. Efficacy of ceftazidime-avibactam salvage therapy in patients with infections caused by Klebsiella pneumoniae carbapenemase-producing $K$. pneumoniae. Clin Infect Dis. 2018;68(3):355-64.
33. Göttig S, et al. Emergence of ceftazidime/avibactam resistance in KPC-3-producing Klebsiella pneumoniae in vivo. J Antimicrob Chemother. 2019;74(11): 3211-16.

34. Munoz-Price LS, et al. Patient-to-patient transmission of KPC variants with reduced ceftazidime-avibactam susceptibility. Antimicrob Agents Chemother. 2019:63(10):e00955-19.

35. Athans V, Neuner EA, Hassouna H, et al. Meropenem-vaborbactam as salvage therapy for ceftazidime-avibactram-resistant klebsiella pneumoniae bacteremia and abscess in a liver transplant recipient. Antimicrob Agents Chemother. 2019;63(1):1-8. https://doi.org/10.1128/ AAC.01551-18. 